\title{
A Method for Evaluating Humanoid Robots Using Anthropometric and Biomechanical Data
}

James Holley, Nicholas Thoma, William K. Verdeyen

NASA Johnson Space Center

james.j.holley@nasa.gov

November 14, 2017 


\section{Existing Human Factors Guidelines}

\section{NASA Constellation Program Human-Systems Integration Requirements (CxP-HSIR)}

- Uses human metrics to drive spacecraft design

- Defines requirements for radiation, safety, waste management, anthropometry, range of motion, mass properties, and strength

- Gives us a way to reverse engineer how to evaluate a robot that will operate well on a spacecraft.

\section{NASA Human Integration Design Handbook (HIDH)}

- Cites CxP-HSIR and provides criticality levels for strength metrics

\section{Criticality Definitions}

- Criticality 1: Requirement for activity related to crew safety.

- Criticality 2: Requirement for activity related to loss of mission. 


\section{Anthropometry}

- Measurement: Body Sizes \& Proportions

- Rationale

- Fit through passageways

- Reach controls, displays, \& equipment

- Human data

- 59 CxP-HSIR measurements

- Valkyrie data

- Robot model

- Physical measurements

- Passes 35 of 59 (59\%) measurements

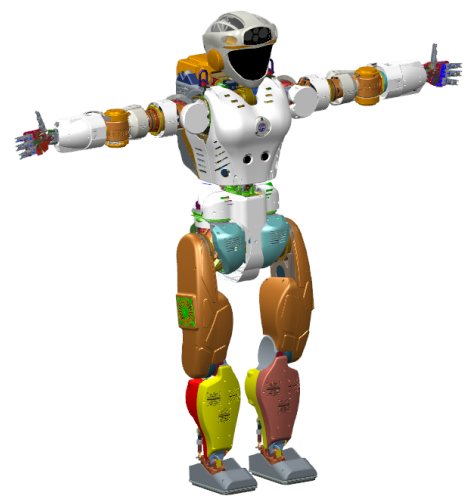




\section{Range of Motion}

- Measurement: Joint Degrees of Freedom

- Rationale

- Interact with controls, tools, \& equipment

- Sufficient reach mobility

- Human data

- 25 CxP-HSIR movements

- Valkyrie data

- Joint limits in robot model

- Passes 16 of 25 (64\%) movements

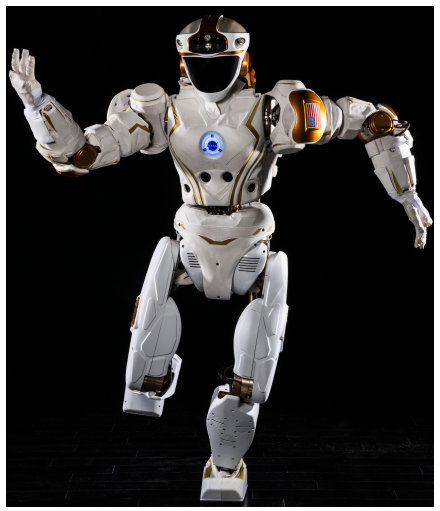




\section{Body Mass}

- Measurement: Whole-Body and Body-Segment Masses

- Rationale

- Weight rating for seats, brackets, \& restraints

- Likelihood of damaging equipment

- Human data

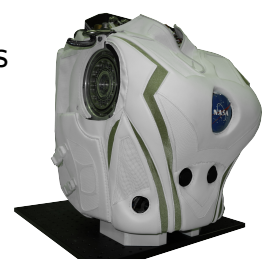

- 12 body-segment, 3 combined body-segment, \& 1 whole-body CXP-HSIR mass

- Valkyrie data

- Center of mass table measurements

- Passes 2 of 16 (13\%) masses

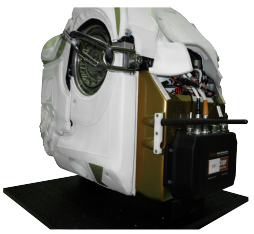




\section{Strength}

- Measurement: Movement Force Output

- Rationale

- Operating force for controls, tools, \& equipment

- Human data

- 34 CxP-HSIR movements

- Valkyrie data

- 6-axis load cell, base, \& handle attachments

- Passes 12 of 34 (35\%) crit. 1 forces

- Passes 11 of $34(\mathbf{3 2 \%})$ crit. 2 forces

- Passes 5 of 34 (15\%) normal operating forces

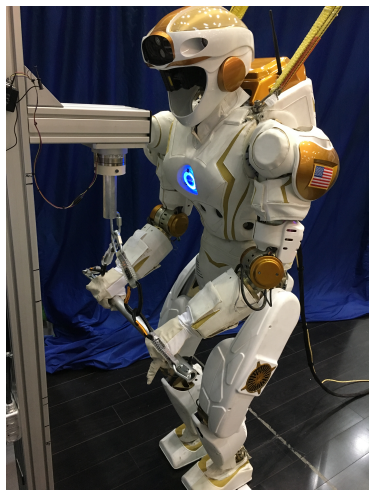




\section{Questions?}
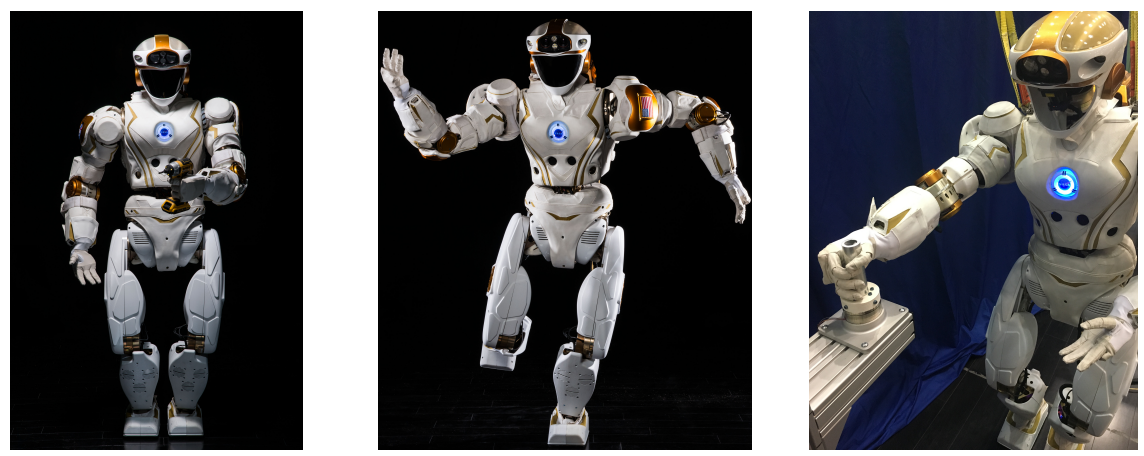